\title{
Roles of YcfR in Biofilm Formation in Salmonella Typhimurium ATCC 14028
}

\author{
Seul I Kim ${ }^{1}$ and Hyunjin Yoon ${ }^{2+}$ \\ ${ }^{1}$ Department of Molecular Science and Technology, Ajou University, Suwon 16499, Republic of Korea; and ${ }^{2}$ Department of \\ Applied Chemistry and Biological Engineering, Ajou University
}

Accepted 16 December 2018.

\begin{abstract}
An increasing number of foodborne diseases are currently attributable to farm produce contaminated with enteric pathogens such as Salmonella enterica. Recent studies have shown that a variety of enteric pathogens are able to colonize plant surfaces by forming biofilms and thereby persist for long periods, which can subsequently lead to human infections. Therefore, biofilm formation by enteric pathogens on plants poses a risk to human health. Here, we deciphered the roles of YcfR in biofilm formation by Salmonella enterica. YcfR is a putative outer membrane protein associated with bacterial stress responses. The lack of YcfR facilitated the formation of multicellular aggregates on cabbage leaves as well as glass surfaces while reducing bacterial motility. $y c f R$ deletion caused extensive structural alterations in the outer membrane, primarily in lipopolysaccharides, outer membrane proteins, cellulose, and curli fimbria, thereby increasing cell surface hydrophobicity. However, the absence of YcfR rendered Salmonella susceptible to stressful treatments, despite the increased multicellular aggregation. These results suggest that YcfR is an essential constituent of Salmonella outer membrane architecture and its absence may cause multifaceted structural changes, thereby compromising bacterial envelope integrity. In this context, YcfR may be further exploited as a potential target for controlling Salmonella persistence on plants.
\end{abstract}

Plants have been generally presumed not to provide favorable habitats for enteric pathogens because of their limited availability of nutrients, fluctuating temperatures during the day and night, atmospheric oxygen on plant surfaces, and antimicrobial compounds released by plants and indigenous microorganisms (Yaron and Römling 2014). However, although enteric pathogens have evolved to adapt to the milieu of host animals and colonize their intestinal tract, they are excreted back to the soil and water through the feces of infected animals

${ }^{\dagger}$ Corresponding author: H. Yoon; yoonh@ajou.ac.kr

Funding: This research was supported by the National Institute of Food and Drug Safety Evaluation (14162MFDS972), and the Basic Science Research Program through the National Research Foundation of Korea (NRF) funded by the Ministry of Science, ICT \& Future Planning (NRF2018R1A2B6007304).

*The $\boldsymbol{e}$-Xtra logo stands for "electronic extra" and indicates that one supplementary table, one supplementary dataset, and eight supplementary figures are published online.

The author(s) declare no conflict of interest.

๑) 2019 The American Phytopathological Society and eventually encounter plants. In this context, farm produce is a reservoir for supporting the persistence of enteric bacteria and also a potential vehicle for transmitting them to their preferable animal hosts (Ochman and Groisman 1995). A worst-case scenario associated with plants contaminated with enteric pathogens is that fresh and fresh-cut produce are typically consumed without thermal treatment and, thus, are likely to cause severe foodborne illnesses. Salmonella enterica and Escherichia coli, the major species accounting for large foodborne outbreaks associated with the consumption of farm produce, were able to survive more than 100 days on green leafy vegetables without provoking disease symptoms such as leaf spots and wilting (Kisluk and Yaron 2012; Markland et al. 2013). Even a small population of enteric pathogens, as little as a few cells, can still cause serious illnesses (Kisluk and Yaron 2012). The ability of enteric pathogens to internalize within a variety of plant tissue types further poses a significant health threat to food processing and storage environments (Deering et al. 2012). To cope with this challenge by enteric pathogens, extensive research efforts have been made in the last decade to understand the strategies of enteric pathogens for surviving in unfavorable plant environments as well as the interactions between enteric pathogens and plants or their natural symbiotic microorganisms, including commensals and parasites.

The ability to form biofilms is one of the most critical determinants required for bacterial adaptation to hostile environments such as plants. Biofilms are an aggregate of microorganisms in which cells adhere to each other on biotic or abiotic surfaces encased within a self-produced matrix of extracellular polymeric substances (EPS) (Hall-Stoodley et al. 2004) that are mainly composed of extracellular DNA and RNA, proteins, and polysaccharides (Danese et al. 2000). In terms of bacterial persistence in plant milieu, the dense biofilm structures provide protective effects against UV radiation, dehydration, detergents, and disinfectants in the field, postharvest storage, or food processing, thereby increasing bacterial resistance under stressful conditions (Lapidot et al. 2006; Mah and O'Toole 2001; Scher et al. 2005). S. enterica and E. coli readily attach to diverse plant tissues such as blossoms, leaves, fruit, and roots and then form irreversible biofilms in a variety of plants (Schikora et al. 2012; Yaron and Römling 2014; Zheng et al. 2013). Extensive studies have been performed to define the bacterial factors responsible for biofilm formation in plant tissues. For example, S. enterica and E. coli possess a diversity of fimbrial and nonfimbrial adhesins on their cell surfaces and utilize them to adhere to plants. The roles of type 1 , curli, and long polar fimbriae in biofilm formation have been investigated in many different $S$. enterica and $E$. coli serotypes and in diverse plants (Schikora et al. 2012; Yaron and Römling 2014). Additionally, the abilities to produce YcfR 
(putative membrane protein), BapA (secreted large surface protein), cellulose, colanic acid, flagella, O-antigen capsule, lipopolysaccharide (LPS), and type 3 secretion systems, all of which make up the surface structures of bacterial cells, were also associated with stable and substantial biofilm formation (Steenackers et al. 2012; Yaron and Römling 2014).

However, it is important to note that, even though many bacterial factors are involved in biofilm formation, their influences to biofilm formation vary in different plants and bacterial serotypes or strains (Schikora et al. 2012; Steenackers et al. 2012; Yaron and Römling 2014). For example, the lack of FliC, a flagella subunit, compromised the adhesion ability to plant leaves in $S$. Senftenberg and E. coli O157:H7 (Berger et al. 2009; Xicohtencatl-Cortes et al. 2009) but not in $S$. Typhimurium (Berger et al. 2009). Furthermore, the deletion of fliC in $S$. Senftenberg showed different biofilm formation abilities depending on the tested plant tissues: a fivefold reduction in attachment to basil leaves (Berger et al. 2009) but no effect in tomato fruit (Shaw et al. 2011). In the cases of curli fimbriae and structural cellulose of EPS, their absence attenuated the attachment of $S$. Enteritidis to alfalfa sprouts (Barak et al. 2007) but hardly influenced the binding of $S$. Typhimurium to parsley leaves (Lapidot et al. 2006). YcfR also exhibited differential effects on biofilm formation of enteric pathogens to plants. YcfR is known to be expressed under diverse stressful conditions in enteric pathogens and is regarded as a multiple stress resistance protein (Zhang et al. 2007). Deletion of $y c f R$ increased biofilm formation and also changed the surface hydrophobicity in E. coli K-12 (Zhang et al. 2007). However, the deletion in $S$. Typhimurium LT2 decreased bacterial attachment to spinach leaves and graph tomato (Salazar et al. 2013). Its role in chlorine resistance was also controversial between two strains of $E$. coli $\mathrm{O} 157: \mathrm{H} 7$, such that there was a positive correlation in Sakai but no correlation in TW14359 (Deng et al. 2011).

We found that $y c f R$ was significantly induced upon contact with diverse plants, including cabbage and cucumber, in several $S$. enterica isolates, implying that its role was associated with bacterial survival in plants. Considering the differential effects of YcfR on biofilm formation and stress resistance among bacterial serotypes and strains, we cannot simply deduce the function of YcfR in S. enterica in contact with plants. Therefore, in this study, we deleted $y c f R$ in $S$. Typhimurium 14028, a representative $S$. enterica strain whose pathogenicity has been extensively studied, and characterized the mutant strain under diverse environments.

\section{RESULTS}

Influence of YcfR on the attachment of $S$. Typhimurium 14028 to plants.

The $y c f R$ gene and its homologs were found to be induced in response to various physical and chemical environmental stimuli (Salazar et al. 2013; Zhang et al. 2007). In accordance with previous reports, we also observed that many $S$. enterica strains, including $S$. Typhimurium 14028 (Fig. 1A), and food or clinical isolates (Supplementary Fig. S1) increased mRNA levels of $y c f R$ when they were exposed to plant tissues. This result suggests that the role of YcfR might be required for Salmonella spp. to adapt to environmental changes encountered in plant tissues. To test this possibility, the $y c f R$ gene was deleted in the genome of $S$. Typhimurium 14028, a well-studied reference strain, and the numbers of live planktonic bacteria were compared between broth media supplemented with or without cabbage. The addition of cabbage leaves stimulated bacterial proliferation (Fig. 1B; Supplementary Fig. S2), suggesting that cabbage tissues might exude nutrient compounds beneficial to Salmonella growth. Interestingly, a deletion of $y c f R$ attenuated the proliferation of planktonic bacteria only in the presence of cabbage, indicating a positive role of YcfR in Salmonella adaption to cabbage-supplemented environments.

The role of YcfR is associated with bacterial attachment to surfaces, resulting in surface-adherent multicellular aggregation (Salazar et al. 2013; Zhang et al. 2007); therefore, the ability of the $\Delta y c f R$ mutant to adhere to plant tissues was evaluated. Bacterial strains were incubated with cabbage leaves statically to promote bacterial adherence to plant surfaces, and bacteria adhering to the surfaces were then counted (Fig. 1C; Supplementary Fig. S3). Salmonella strains lacking ycfR adhered to cabbage leaves approximately 2.5 -fold more than that on wild-type Salmonella at $8 \mathrm{~h}$. This result was contradictory to a previous study showing the positive effect of YcfR on bacterial attachment to spinach leaves in $S$. Typhimurium LT2 (Salazar et al. 2013). Although $S$. Typhimurium 14028 and $S$. Typhimurium LT2 are closely related at the genomic level, bacterial physiological characteristics such as biofilm formation ability might be different between them, as demonstrated in many other cases (Barak et al. 2007; Berger et al. 2009; Deng et al. 2011; Lapidot et al. 2006). In subsequent experiments of this study, the roles of YcfR were defined in $S$. Typhimurium 14028 by focusing on the bacterial ability to adhere to surfaces or each other, as well as to address the discrepancy in bacterial attachment to plants between the two Salmonella strains 14028 and LT2.
A

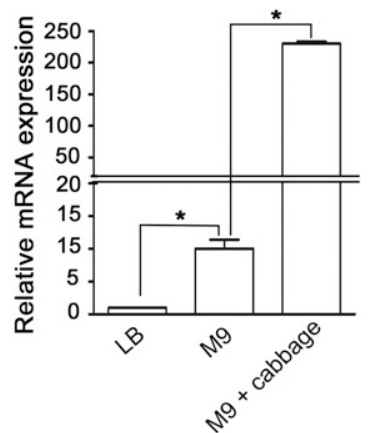

B

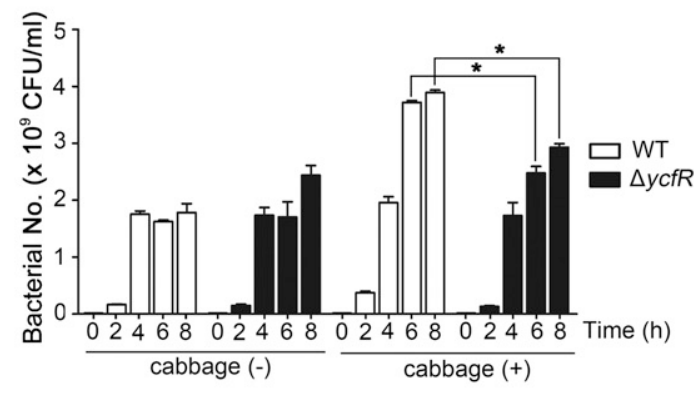

C

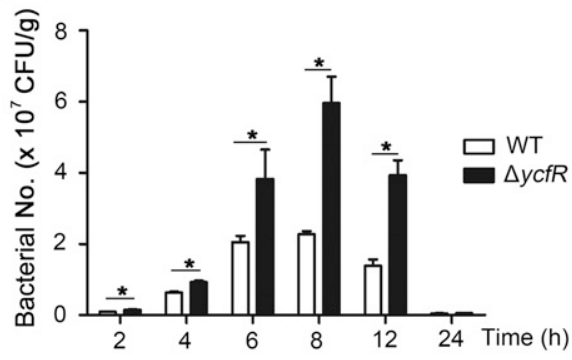

Fig. 1. Influence of $y c f R$ in Salmonella adaptation to cabbage-supplemented conditions. A, Relative $y c f R$ mRNA levels in different growth conditions. Expression of $y c f R$ in Luria-Bertani (LB) broth was set to 1.0. B, Viability of wild-type (WT) and $\Delta y c f R$ strains in M9 minimal medium broth supplemented with or without cabbage. $\mathrm{pH}$ measurements were conducted in parallel. C, Attachment of WT and $\Delta y c f R$ strains to cabbage surfaces. Differences with $P$ values $<0.05$ are marked with asterisks. 
Increased biofilm-like cell aggregates by the lack of YcfR in $S$. Typhimurium 14028 .

In order to clarify the role of YcfR in biofilm formation in $S$. Typhimurium 14028, bacterial strains were incubated in M9 minimal medium broth in glass tubes statically, and biofilms that formed on the surface were scored by staining with crystal violet, which is retained in peptidoglycan layers of bacterial cell walls, thereby indicating the presence of cells (Fig. 2A). The absence of YcfR increased biofilm formation more than threefold in the 14028 strain, and the introduction of a plasmid expressing $y c f R$ (pYcfR) completely restored the level of biofilm formation to that of wild-type bacteria. On the other hand, YcfR did not influence biofilm formation in $S$. Typhimurium LT2 (Supplementary Fig. S4). Given that biofilm is a consequential behavior in response to environmental stimuli, the role of YcfR in biofilm formation might be altered under different growth conditions. However, when brain-heart infusion (BHI) broth was used instead of M9 minimal medium broth, the lack of YcfR did not significantly influence biofilm formation in both of the 14028 and LT2 strains. This discrepancy in biofilm formation abilities between the 14028 and LT2 strains might be attributable to differences other than $y c f R$ in the structure and organization of their genome sequences (as described in Discussion). Biofilm behaviors are also predictable by staining culri or polysaccharide elements using bacteriological dyes such as Congo red and Calcofluor white. Congo red stains extracellular matrices consisting of culri fimbriae and cellulose and determines bacterial red, dry, and rough (rdar) morphotypes (Römling 2005), while Calcofluor white specifically binds to extracellular cellulose moiety (Paytubi et al. 2017). The rdar morphotypes and Calcofluor fluorescence phenotypes were comparable again between wild-type and $\Delta y c f R$ mutant strains
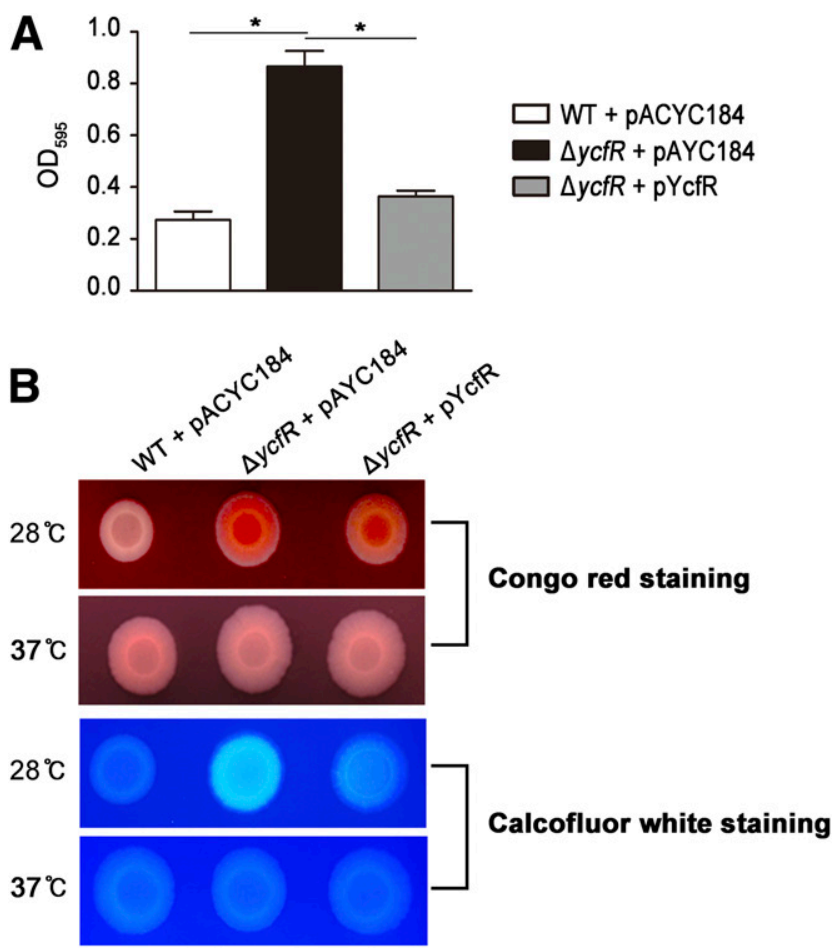

Fig. 2. Biofilm formation comparison between wild-type (WT) and $\Delta y c f R$ strains in Salmonella Typhimurium 14028. A, Biofilm staining using crystal violet. Asterisks indicate differences with $P$ values $<0.05$. B, Congo red and Calcofluor white staining. Production of culri fimbriae and cellulose was examined using red, dry, and rough morphotyping (top) and cellulose staining (bottom) at 28 and $37^{\circ} \mathrm{C}$. Bacterial strains were transformed with pYcfR or pACYC184. in $S$. Typhimurium LT2, both of which showed rdar colonies on Congo red agar plates and fluorescent colonies on Calcofluor white agar plates at two different temperatures. However, the lack of YcfR intensified the red color on Congo red agars in $S$. Typhimurium 14028 at $28^{\circ} \mathrm{C}$, indicating increased biofilm formation in the $\Delta y c f R$ mutant strain (Fig. 2B). Calcofluor white staining also exhibited strong fluorescence in the $\Delta y c f R$ mutant strain at $28^{\circ} \mathrm{C}$, suggesting increased cellulose production by the absence of $y c f R$ in $S$. Typhimurium 14028 .

\section{Alteration in the outer membrane structure by the lack of YcfR.}

The outer membrane of gram-negative bacteria is an asymmetric bilayer balanced between an inner leaflet of phospholipids and an outer leaflet of LPS, and decorated with diverse proteins in the form of integral membrane proteins or membraneanchored proteins. Given that $y c f R$ encodes an outer membrane protein based on its amino acid sequence alignment analysis, its absence might cause alterations in structural compositions of the outer membrane, leading to enhanced cellular aggregation. Therefore, we investigated whether the lack of YcfR could influence the structural compositions of outer membrane proteins and LPS, the two main constituents of the outer membrane.

Outer membrane proteins, which make up approximately $50 \%$ of the outer membrane mass, were purified and their profiles were compared between wild-type and $\Delta y c f R$ mutant strains. Deletion of $y c f R$ resulted in differential expression in a certain number of outer membrane proteins of $S$. Typhimurium 14028 , as indicated by the black and white arrowheads in Figure $3 \mathrm{~A}$. However, the lack of YcfR did not influence protein compositions significantly in the outer membrane of $S$. Typhimurium LT2 (Supplementary Fig. S5).

LPS is a large amphipathic polymer consisting of three moieties, including lipid A, core oligosaccharides, and $\mathrm{O}$ antigen, and plays critical roles not only in protecting cells from harsh environments such as antibiotics and host immune systems but also in forming surface-adherent multicellular communities such as biofilms (Whitfield and Trent 2014; Zhang et al. 2013). LPS banding profiles were compared between wild-type and $\Delta y c f R$ mutant strains, and a shift in the LPS ladder rungs was used as an indicator of LPS modifications (Fig. 3B). Deletion of $y c f R$ caused shifts of multiple LPS bands and also increased the intensity of bands in presumably the lipid A-core moiety in $S$. Typhimurium 14028, indicating structural modifications in LPS components. However, deletion of $y c f R$ in $S$. Typhimurium LT2 did not result in significant modifications
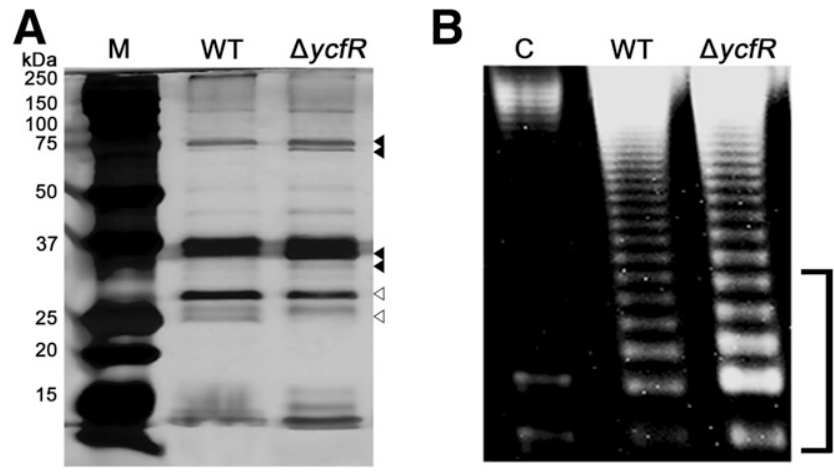

Fig. 3. Altered outer membrane structures by the lack of YcfR. A, Sodium dodecyl sulfate polyacrylamide gel electrophoresis (PAGE) analysis of outer membrane proteins. Black and white arrowheads indicate proteins increased and decreased in $\Delta y c f R$, respectively. Lane $\mathrm{M}$ indicates size markers and WT = wild type. B, Sodium deoxycholate-PAGE analysis of lipopolysaccharide (LPS). C indicates Escherichia coli O55:B5 LPS as a control. A right square bracket shows structural modifications in LPS components by the lack of $y c f R$. 
in LPS profiles, indicating differential LPS compositions by the lack of YcfR between 14028 and LT2. Taken together, these results demonstrated that $S$. Typhimurium 14028 lacking YcfR underwent remarkable changes in the outer membrane structures.

\section{Increased surface hydrophobicity by the lack of YcfR.}

$S$. Typhimurium 14028 lacking YcfR showed alterations in the structural constituents of outer membrane proteins and LPS. The structural alteration and modification in the outer membrane might cause physiological changes in bacterial surfaces, which could facilitate bacterial adhesion and attachment to biotic or abiotic surfaces. For successful biofilm formation, electrostatic repulsion between bacteria and surfaces, either environmental surfaces or other bacterial cell surfaces, should be overcome at the early phase of biofilm formation. Then, hydrophobic functional moieties aligned on bacterial surfaces accelerate bacterial attachment to surfaces. In an autoaggregation assay, we observed that the lack of YcfR promoted multicellular aggregation in $S$. Typhimurium 14028 and the introduction of $\mathrm{pYcfR}$ complemented the $\Delta y c f R$ strain, restoring an aggregation level to that of the wild-type strain (Fig. 4A; Supplementary Fig. S6). In accordance with this observation, the hydrophobicity of bacterial cell surfaces was also increased by the lack of YcfR, even though the introduction of pYcfR could not sufficiently complement the altered hydrophobicity in the $\Delta y c f R$ strain (Fig. 4B). The physiological changes caused by the lack of YcfR, including increases in cell surface hydrophobicity and autoaggregation, corroborated the biofilm behavior of the $\Delta y c f R$ strain

Curli fimbriae and cellulose: Potential adhesion determinants associated with YcfR.

The structural compositions of bacterial biofilm vary, depending on the environmental conditions. The major components associated with Salmonella biofilm are divided into proteinaceous compounds and EPS (Steenackers et al. 2012; Yaron and Römling 2014). The main proteinaceous compounds are fimbriae such as curli, type 1, long polar, and plasmid-encoded fimbriae. The EPS fraction includes cellulose, colanic acid, O-antigen capsule, and LPS. In order to define the bacterial determinants responsible for biofilm behavior in the $\Delta y c f R$ strain, the expression levels of genes related to biofilm formation were compared between the wild-type and $\Delta y c f R$ strains. The lack of YcfR led to tremendous increases in the expression of curli genes, including the master regulator $\operatorname{csg} D$ as well as the $\operatorname{csg} B A C$ and $\operatorname{cgs} E F G$ operons (Fig. 5A), which is indicative of abundant production and localization of curli fimbriae on the surfaces of the $\triangle y c f R$ strain. The increased expressions of $c s g D E G F$ and $c s g B A C$ in the $\triangle y c f R$ strain were partially complemented by the production of YcfR from pYcfR. On the other hand, genes required for the production of O-antigen capsule (yihQO) and colanic acid (wza, wzc, and $w c a A D$ ) did not show significant differences in their expression depending on the presence of YcfR, while cellulose genes (bcs $A B C$ ) and type 1 fimbriae genes (fimZ and fimIF) were downregulated in the $\Delta y c f R$ strain (Fig. 5A; Supplementary Fig. S7). Downregulation of $b c s A B C$ genes by the lack of $y c f R$ was implausible, because the Calcofluor white staining result suggested an increase in cellulose production (Fig. 2B). However, cellulose production can be regulated at a posttranscriptional level by bis- $\left(3^{\prime}-5^{\prime}\right)$-cyclic dimeric GMP (c-di-GMP), a secondary messenger molecule synthesized by di-guanylate cyclases. c-di-GMP binds to BcsA and BcsB and promotes their conformational alteration to activate cellulose production (Mayer et al. 1991; Morgan et al. 2014). We observed that the lack of $y c f R$ increased not only the expression of $a d r A$, a primary gene encoding di-guanylate cyclase (Ahmad et al. 2011;
Kader et al. 2006; Steenackers et al. 2012), but also the production of c-di-GMP (Fig. 5B). These results suggest that increases in curli fimbriae and cellulose production might be predominantly responsible for the enhanced biofilm development in the $\Delta y c f R$ strain. The CsgD and c-di-GMP regulatory network is coordinated by RpoS, a global transcriptional regulator responsible for bacterial adaptation to diverse stressors (Brown et al. 2001; Römling et al. 1998; Weber et al. 2006). Considering the increased rpoS expression in the $\Delta y c f R$ strain (Fig. 5A), the altered outer membrane structure due to the lack of YcfR might be sensed by RpoS, which might then induce the coordinated biofilm behavior (as suggested in Discussion).

Bacterial interactions with other cells and surfaces can also be influenced by bacterial motility. When bacterial motility was compared on agar plates, deletion of $y c f R$ profoundly retarded bacterial motility in $S$. Typhimurium 14028, and the defective motility was complemented with the introduction of pYcfR (Fig. 6A). In line with the decreased motility of the $\Delta y c f R$ strain, the expression levels of flagella master operon flhDC and those of $f l i C$ and $f l j B$ encoding two different types of flagella filament proteins were downregulated by the deletion of $y c f R$ in the 14028 strain (Fig. 6B). The impaired motility by the lack of $y c f R$ might also be integrated into the regulatory network encompassing RpoS and c-di-GMP. RpoS represses the transcription of flagella master operon $f l h D C$, and c-di-GMP coupled with YcgR inhibits bacterial motility by binding directly to flagella motor proteins (Paul et al. 2010; Uchiyama et al. 2010).

In conclusion, the multifaceted phenotypes by the lack of YcfR, including the increased expression in curli fimbriae genes, the increased cellulose production, and the decreased motility, were consistent with the enhanced bacterial aggregation on biotic and abiotic surfaces in the $\Delta y c f R$ strain.

\section{Importance of YcfR in the maintenance} of cell envelope integrity.

The cell envelope determines the bacterial shape and provides a protective interface against a myriad of environmental threats such as disinfectants and antimicrobial compounds.

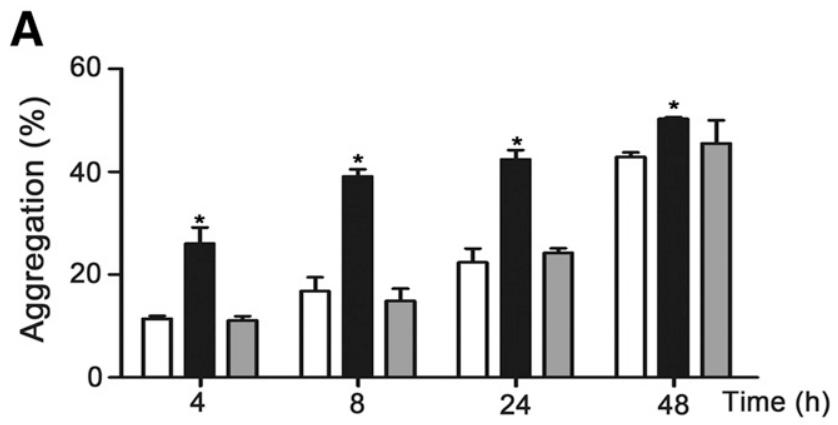

B

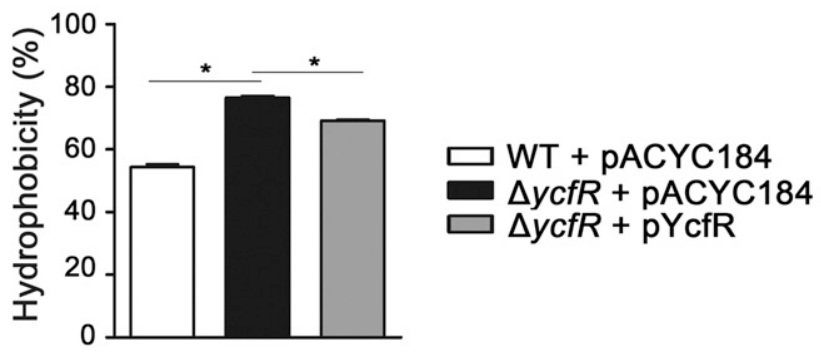

Fig. 4. Alterations in physiological properties by the lack of YcfR in Salmonella Typhimurium 14028. A, Autoaggregation assay. Asterisks indicate different values from those of the wild-type (WT) strains with $P$ values $<0.05$. B, Hydrophobicity assay. Asterisks indicate differences with $P$ values $<0.05$ 
Therefore, the maintenance of cell envelope integrity is critical for bacterial viability. YcfR has been predicted to be an outer membrane protein and its roles related to bacterial resistance have been studied in several pathogenic bacteria (Deng et al. 2011; Salazar et al. 2013; Zhang et al. 2007). Enteric pathogens devoid of $y c f R$ and its homologs became vulnerable to treatments of acid, heat, hydrogen peroxide, chlorine, and cadmium. However, given the increased biofilm-like cell aggregates in the $\Delta y c f R$ strain, these previous observations are debatable because biofilm is supposed to provide an ecological defense niche for bacteria under unfavorable conditions. We observed that deletion of $y c f R$ caused profound structural and physiological changes in the Salmonella cell envelope. Imbalanced structural compositions due to the lack of YcfR may compromise the integrity of cell envelope structure, thereby threatening bacterial vitality. To examine the influence of YcfR on cell envelope integrity, outer membrane permeability was compared between wild-type and $\Delta y c f R$ strains by measuring the accessibility of the hydrophobic fluorescent probe N-phenyl-1-naphthylamine (NPN) to the outer membrane (Fig. 7A). Salmonella strain slacking YcfR increased the accumulation of NPN, indicating structural perturbations to the outer membrane. The increased outer membrane permeability to hydrophobic NPN might be partially attributable to the increased cell surface hydrophobicity of the $\Delta y c f R$ strain (Fig. 4B). Altered permeability of the outer membrane due to the lack of YcfR also influenced bacterial resistance to acid treatments. Organic acids penetrating bacterial envelope structures have been reported to cause a reduction in the cellular $\mathrm{pH}$ and inhibit cellular metabolism and transport systems, consequently leading to cell death (Raybaudi-Massilia et al. 2009). Many different kinds of organic acids approved as "generally recognized as safe" are used to prevent food spoilage from microorganisms, including citric, lactic, acetic, and sorbic acids. Deletion of $y c f R$ rendered $S$. Typhimurium 14028 more susceptible to citric acid and lactic acid treatments, suggesting that the increased membrane permeability by the lack of YcfR resulted in increased internalization of acids across the outer membrane (Fig. 7B). In addition, $S$. Typhimurium 14028 lacking $y c f R$ also became more susceptible to hydrogen peroxide and sodium hypochlorite, both of which are generally used for sanitizing raw fruit and vegetables during the washing or peeling process (Rico et al. 2007). Bacterial resistance against stressful conditions also varied between 14028 and LT2 strains, as observed previously (Fornefeld et al. 2017; Lee et al. 1995). S. Typhimurium strain LT2 was more vulnerable to acid treatments and the lack of YcfR did not influence bacterial tolerance to acid treatments, whereas its absence attenuated bacterial resistance against hydrogen peroxide and sodium hypochlorite treatments (Supplementary Fig. S8).

\section{DISCUSSION}

$S$. Typhimurium is a foodborne pathogen that can survive inside hosts and also persist in food environments. This lifestyle of $S$. Typhimurium requires diverse strategies to circumvent variable hostile environments. Biofilm formation is a representative defense strategy to resist against unfavorable stressors. Salmonella spp. are capable of systematically modulating the expression of groups of genes in response to environmental stimuli and alternate growth modes between free-living motile cells and members of an aggregated multicellular community in a biofilm. A variety of structural appendages distributed on the bacterial cell surface are associated with biofilm formation. These bacterial biofilm determinants include proteinaceous compounds of fimbriae, flagella, and diverse outer membrane proteins and EPS compounds, including cellulose, LPS, colonic acid, and capsule. Coordinated regulation of these bacterial structural constituents in response to external stimuli suggests the presence of a master signaling molecule that orchestrates bacterial fitness depending on environments. In Salmonella and other bacteria, the transcriptional regulator $\mathrm{CsgD}$ is positioned in the center of a complex regulatory circuit that determines bacterial behaviors between planktonic and sessile modes (Chambers and Sauer 2013). Multiple extracellular stimuli, including starvation, osmolarity, and cell envelope stress, are integrated into $\mathrm{CsgD}$ via global regulators such as RpoS, OmpR, and RcsB. CsgD, in turn, activates the production of curli fimbriae while repressing the expression of several flagellar biosynthesis genes. In addition to CsgD, c-di-GMP functions as a secondary messenger for controlling bacterial transition between motility and sessility (Simm et al. 2004). High concentrations of c-di-GMP increase the production of curli fimbriae by the $\operatorname{csg} B A C$ operon and cellulose by the $b c s A B Z C$ operon via transcriptional or posttranscriptional mechanisms to stimulate biofilm formation (Kader et al. 2006; Steenackers et al. 2012). The levels of c-di-GMP are modulated by a complex network consisting of multiple c-di-GMPsynthesizing (di-guanylate cyclase; GGDEF domain protein) and c-di-GMP-degrading (phosphodiesterase; EAL domain protein) proteins. $S$. Typhimurium possesses 22 genes encoding GGDEF or EAL domain proteins in the genome, and AdrA encoded by the $a d r A$ gene is one of the prominent di-guanylate cyclases responsible for c-di GMP production (Ahmad et al. 2011; Kader et al. 2006; Steenackers et al. 2012). Interestingly, we observed that the lack of YcfR significantly increased the transcription of both $\operatorname{csg} D$ and $a d r A$ genes and accordantly increased the production of c-di-GMP, suggesting increased biosynthesis of curli fimbriae and cellulose in the $\Delta y c f R$ strain (Fig. 5). The impaired integrity in the bacterial outer membrane architectures by the lack of YcfR might be sensed
A

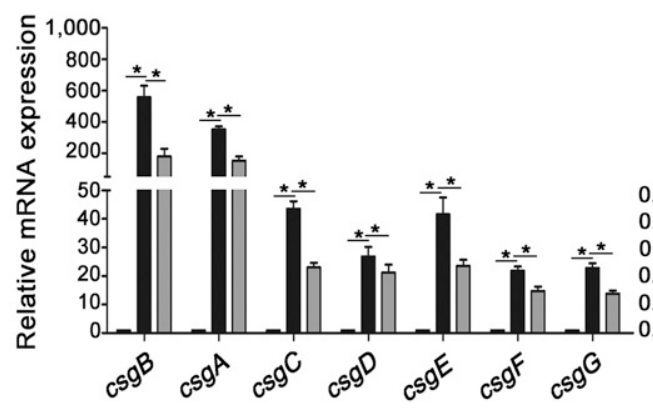

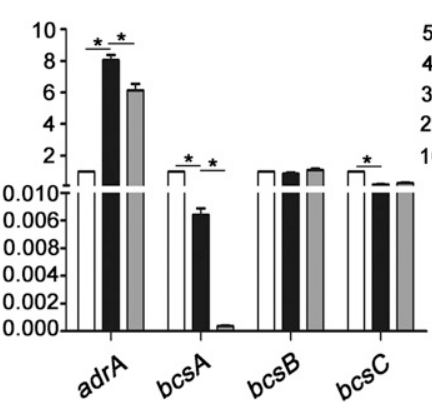

B

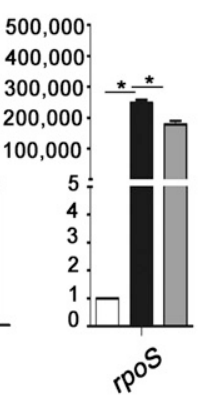

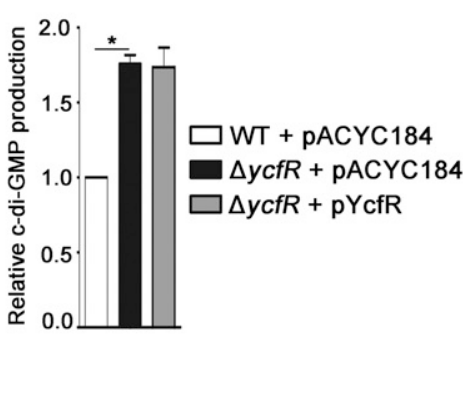

Fig. 5. Influence of YcfR on expression of genes associated with biofilm formation. A, Relative expressions of genes associated with biofilm in the $\Delta y c f R$ strain. The mRNA levels in the wild-type (WT) strains were set to 1.0 for each gene. B, Bis- $\left(3^{\prime}-5^{\prime}\right)$-cyclic dimeric GMP assay. Values with differences of $P$ values $<0.05$ are denoted with asterisks. 
by global transcriptional regulators such as RpoS and OmpR (Gerstel et al. 2003) which might, in turn, coordinate the CsgD and c-di-GMP regulatory network to divert bacterial behavior from a planktonic to biofilm lifestyle (Povolotsky and Hengge 2012).

$S$. Typhimurium 14028 is closely related to $S$. Typhimurium LT2 at the genomic level, with over $98 \%$ sequence identity, yet the phenotypes by the lack of YcfR were significantly different between these two strains. Although both strains showed increased cellular aggregation due to the lack of YcfR, bacterial surface hydrophobicity and attachment abilities to abiotic and biotic surfaces were totally different between them. An $S$. Typhimurium LT2 derivative devoid of YcfR did not alter cell surface hydrophobicity but showed attenuations in attachment to surfaces of glass tube and spinach leaves (Salazar et al. 2013). A plausible explanation for this discrepancy could

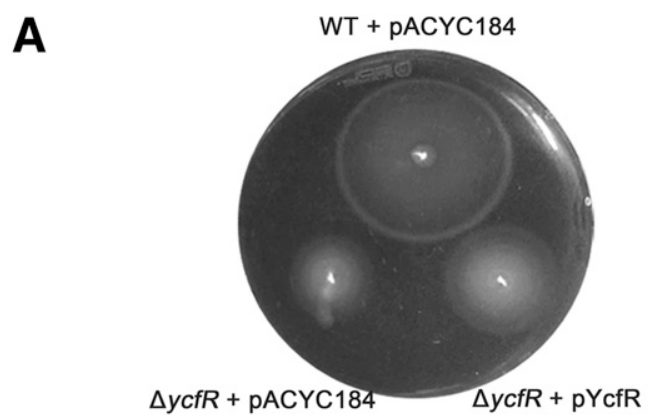

B

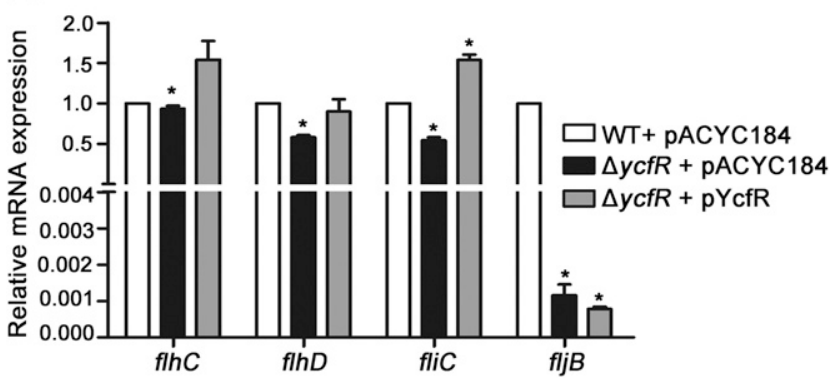

Fig. 6. Influence of YcfR on Salmonella motility. A, Motility assay on agar plates. B, Relative expressions of genes associated with flagella in the $\Delta y c f R$ strain. mRNA levels in the wild-type (WT) strains were set to 1.0 for each gene. Values with differences of $P$ values $<0.05$ in comparison with the WT strain are denoted with asterisks. involve the differences in their structural constituents and compositions of the outer membrane. We observed that the deletion of $y c f R$ caused significant alterations in the composition and abundance of LPSs and outer membrane proteins in $S$. Typhimurium 14028 but not in $S$. Typhimurium LT2 (Fig. 3). The rdar morphotypes and Calcofluor fluorescence phenotypes in the absence of YcfR were also different between these two strains, indicating that the abundance of curli fimbriae and cellulose in $S$. Typhimurium 14028 was also influenced by YcfR but not in $S$. Typhimurium LT2 (Fig. 2B). Different phenotypic responses between the LT2 and 14028 strains to unfavorable stimuli have been observed in several studies. For example, the LT2 strain is less tolerant to stressful conditions such as growth medium changes and acid treatments than the 14028 strain and other strains due to malfunctioning RpoS, which starts with a rare TTG codon and compromises bacterial fitness under hostile environments (Fornefeld et al. 2017; Wilmes-Riesenberg et al. 1997). In addition, in contrast to the 14028 strain, the LT2 strain is highly attenuated in virulence and readily cleared out in host animals, probably due to imperfect RpoS resulting in structural vulnerability to host immune responses (Swords et al. 1997; Wilmes-Riesenberg et al. 1997). RpoS and other global regulators sensing a multitude of environmental stimuli prime bacteria to resist diverse stressors by compositional alterations of membranous components, stimulation of stress resistance, and modulation of virulence and metabolism (Lévi-Meyrueis et al. 2014; Lago et al. 2017). The extensive alterations in the outer membrane structure of $S$. Typhimurium $14028 \Delta y c f R$ strain suggest that the 14028 strain coordinates the constitutional composition of the outer membrane more systematically than the LT2 strain in response to the structural imbalance by the lack of YcfR.

This study defined the roles of YcfR in biofilm formation in $S$. Typhimurium 14028. The lack of YcfR was associated with extensive changes in cell surface structures of the 14028 strain, leading to bacterial aggregation and attachment to biotic or abiotic surfaces. In view of the controversial roles of YcfR in biofilm formation among different bacterial genera and strains, this study provides experimental evidence to show that YcfR is important for $S$. Typhimurium 14028 to maintain the envelope integrity, and its absence results in multicellular aggregation. Furthermore, the attenuated resistance in the absence of YcfR against diverse stressors suggests that YcfR can be a promising target for controlling contamination with enteric pathogens in farm produce.

A

B
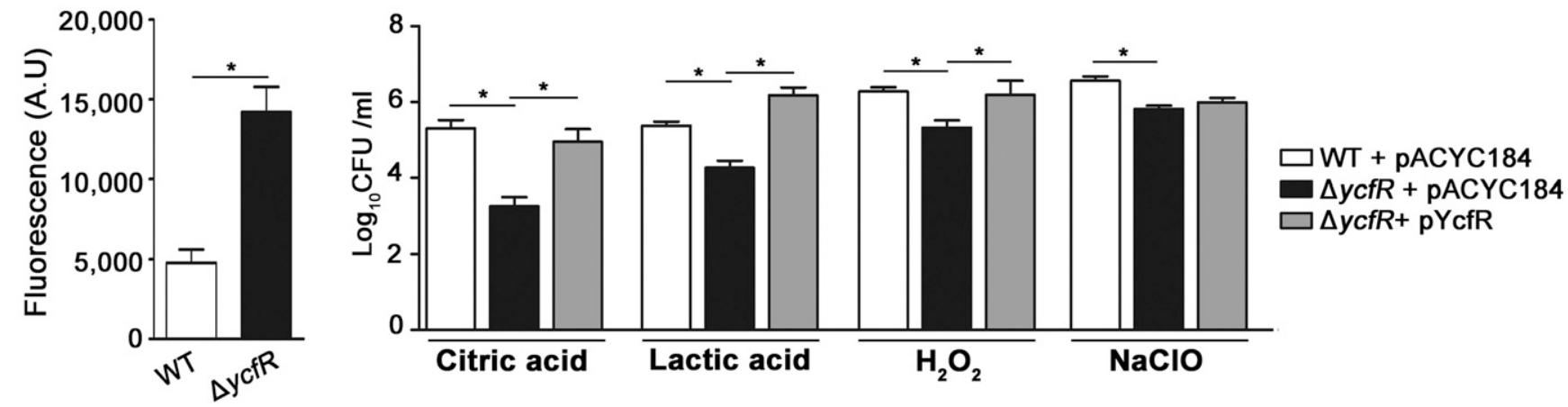

Fig. 7. Assessment of the outer membrane integrity in the absence of YcfR. A, N-phenyl-1-naphthylamine (NPN) uptake assay. NPN associated with bacterial cells was measured at $10 \mathrm{~min}$ after the NPN solution treatment. Fluorescence intensity was normalized using the number of live cells tested. WT = wild type. B, Bacterial resistance against diverse stressful conditions. Cells at a density of $5 \times 10^{8} \mathrm{CFU} / \mathrm{ml}$ were treated with $1.5 \%$ citric acid (pH 2.2 ), $0.1 \%$ lactic acid (pH 3.3), $20 \mathrm{mM}$ hydrogen peroxide, or $0.05 \mathrm{mM}$ sodium hypochlorite and live cells were then counted. Asterisks indicate statistically significant differences with $P$ values $<0.05$. 


\section{MATERIALS AND METHODS}

\section{Bacterial strains and plasmids.}

Two strains of S. enterica subsp. enterica serovar Typhimurium, 14028 (ATCC 14028) and LT2 (ATCC 700720), were used as wild-type strains. For $\Delta y c f R$ strain construction in $S$. Typhimurium 14028, the phage $\lambda$-derived Red recombination system was applied (Datsenko and Wanner 2000). The kanamycin resistance (kan) cassette of pKD13 was amplified using polymerase chain reaction (PCR) with two primers of Red-ycfRF and Red-ycfRR, which were designed to produce PCR product termini to be homologous to flanking regions of $y c f R$ in the chromosome. The PCR product was introduced into $S$. Typhimurium 14028 harboring pKD46 for replacing $y c f R$ with the kan cassette (Datsenko and Wanner 2000). The inserted kan cassette was removed to generate an in-frame deletion of $y c f R$, using the pCP20 plasmid to provide the flip recombinase (Datsenko and Wanner 2000). Deletion of $y c f R$ was verified by diagnostic PCR using two primers of ycfR-DF and ycfR-DR. For $\Delta y c f R$ strain construction in $S$. Typhimurium LT2, the phage P22-mediated transduction method was used (Schmieger 1972). To construct pYcfR, a plasmid expressing $y c f R$ under its own promoter, DNA sequences containing $y c f R$ and its presumable promoter (Zhang et al. 2007) were amplified using primers of ycfR-CF and ycfRCR and cloned into pACYC184. All primers used in this study are listed in Supplementary Table S1.

\section{Growth conditions and cabbage preparation.}

Bacteria were cultured in Luria-Bertani (LB) and BHI media (BD, Sparks, MD, U.S.A.) or M9 minimal medium supplemented with $10 \mathrm{mM}$ glucose and $2 \%$ histidine. Antibiotics were all purchased from Sigma-Aldrich (St. Louis, MO, U.S.A.) and added when required as follows: kanamycin, $50 \mu \mathrm{g} / \mathrm{ml}$; chloramphenicol, $35 \mu \mathrm{g} / \mathrm{ml}$; and ampicillin, $50 \mu \mathrm{g} / \mathrm{ml}$. Cabbage leaves were rinsed with $0.01 \% \mathrm{NaClO}$ and washed twice with double-distilled (dd) $\mathrm{H}_{2} \mathrm{O}$. In cocultivation with bacteria, cabbage leaves were cut into pieces $(2$ by $8 \mathrm{~cm}$ ) and added in flasks or tubes. Bacterial growth and viability were estimated using an optical density at $600 \mathrm{~nm}\left(\mathrm{OD}_{600}\right.$ measurement and colony counting onto XLD agar (Oxoid, Hants, UK), respectively.

\section{Bacterial attachment onto cabbage leaves.}

Salmonella strains grown in M9 minimal medium broth overnight were washed with phosphate-buffered saline (PBS), then added to $45 \mathrm{ml}$ of PBS containing $5 \mathrm{~g}$ of cabbage leaves at a density of $2 \times 10^{7} \mathrm{CFU} / \mathrm{g}$ of cabbage in 50-ml conical tubes, and incubated statically at room temperature (Salazar et al. 2013; Zhang et al. 2007). At the indicated times after incubation, cabbage leaves were removed, dried for $1 \mathrm{~h}$, and rinsed with PBS. Bacteria attached onto cabbage leaves were detached using glass beads by beating and then counted by plating on XLD agar.

\section{Biofilm assay.}

Salmonella strains grown in M9 minimal medium broth or BHI medium broth till midlog phase were used to inoculate fresh $\mathrm{M} 9$ or $\mathrm{BHI}$ broth at $\mathrm{OD}_{600}=1.0$, then statically cultured in glass tubes at $37^{\circ} \mathrm{C}$ for $48 \mathrm{~h}$. Glass tubes were washed using PBS twice, and bacterial cells attached to the glass surfaces were stained using $1 \%$ crystal violet (Sigma-Aldrich) for $15 \mathrm{~min}$. The biofilm was then treated with $33 \%$ acetic acid to extract the dye, and the released dye was measured at $\mathrm{OD}_{595}$.

\section{Congo red-based rdar morphotyping and Calcofluor white staining.}

Bacterial cells were precultured in LB broth deprived of $\mathrm{NaCl}$ till they reached midlog phase, and equivalent numbers of cells resuspended in PBS at $\mathrm{OD}_{600}=1.0$ were spiked onto $\mathrm{LB}$ agar without $\mathrm{NaCl}$ but complemented with Congo red (40 $\mu \mathrm{g} / \mathrm{ml}$; Sigma-Aldrich) and Coomassie brilliant blue (20 $\mu \mathrm{g} / \mathrm{ml}$; Sigma-Aldrich). Agar plates were then incubated at $28^{\circ} \mathrm{C}$.

Calcofluor white staining was performed as described previously (Paytubi et al. 2017), with minor modification. Bacterial cells were cultured and prepared in the same manner described in Congo red staining, except with bacterial suspension at $\mathrm{OD}_{600}=2.0$. Salmonella cells in $\mathrm{PBS}$ at $\mathrm{OD}_{600}=2.0$ were spotted onto $\mathrm{LB}$ agar without $\mathrm{NaCl}$ but with Calcofluor white M2R (10 $\mu \mathrm{g} / \mathrm{ml}$; Sigma-Aldrich) and incubated at $28^{\circ} \mathrm{C}$. Agar plates were observed under UV light (VILBER UV Hand Lamp BVL-8; Lab Unlimited, Surrey, UK).

\section{Autoaggregation assay.}

Salmonella strains were cultured in M9 minimal medium broth and washed with PBS twice. Bacterial cells were resuspended in $5 \mathrm{ml}$ of $\mathrm{PBS}$ at $\mathrm{OD}_{600}=1.0$ (recorded as $\mathrm{A}_{0}$ ), then incubated statically at room temperature. The $\mathrm{OD}_{600}$ was measured from $100 \mu \mathrm{l}$ of the suspension at the top at the indicated time points and recorded as $\mathrm{A}_{\mathrm{i}}$. The autoaggregation (percentage) of bacterial cells was estimated using the formula of $\left[\left(A_{0}-A_{i}\right) / A_{0}\right] \times 100$ (Rahman et al. 2008).

\section{Hydrophobicity assay.}

Bacterial cells grown in M9 minimal medium were washed with PBS twice and resuspended in $2 \mathrm{ml}$ of PBS at $\mathrm{OD}_{600}=1.0$ (recorded as $\mathrm{H}_{0}$ ). The bacterial suspension was mixed vigorously with $800 \mu$ l of xylene (Sigma-Aldrich), then incubated at room temperature for $1 \mathrm{~h}$. The turbidity of the aqueous phase was measured at $\mathrm{OD}_{600}$ and recorded as $\mathrm{H}$. The hydrophobicity of bacterial surfaces was calculated as follows: hydrophobicity $(\%)=\left[\left(\mathrm{H}_{0}-\mathrm{H}\right) / \mathrm{H}_{0}\right] \times 100$ (Gómez Zavaglia et al. 2002)

\section{c-di-GMP assay.}

Bacterial cells cultured on $\mathrm{LB}$ agar without $\mathrm{NaCl}$ at $28^{\circ} \mathrm{C}$ for $48 \mathrm{~h}$ were scraped, resuspended in $\mathrm{PBS}$, and subjected to $\mathrm{OD}_{600}$ measurement for normalizing cell amounts. Cells were then centrifuged, resuspended in $0.19 \%$ formaldehyde solution, and incubated on ice for $10 \mathrm{~min}$. Bacterial cells were centrifuged, washed with distilled water twice, and resuspended in distilled water. The intracellular c-di-GMP was extracted by heat and ethanol precipitation method (Roy et al. 2013; Simm et al. 2009). The cells were heated at $100^{\circ} \mathrm{C}$ for $5 \mathrm{~min}$ and ethanol was added to the cells suspension at a concentration of $70 \%$. Bacterial cells were homogenized using a 20-gauge needle and incubated on ice for $10 \mathrm{~min}$. Cells lysate was centrifuged at $17,000 \times g$ for $10 \mathrm{~min}$ and the supernatant containing c-di-GMP was dried using a 1-liter rotary evaporator (EYELA, Bohemia, NY, U.S.A.) to remove the ethanol solution. The sample was frozen at $-80^{\circ} \mathrm{C}$ over $1 \mathrm{~h}$, then was lyophilized with a TFD5503 Bench-Top freezedryer (ilShin Biobase Europe, Gelderland, Netherlands). c-di-GMP in the lyophilized sample was quantified using a c-di-GMP enzyme-linked immunosorbent assay kit (EIAab, Hubei, China) according to the manufacturer's instructions.

\section{Motility assay.}

Bacteria were cultured in LB broth till midlog phase and resuspended in $\mathrm{PBS}$ at $\mathrm{OD}_{600}=1.0$. Bacterial suspensions of equivalent volumes were injected into soft LB agar plates containing $0.3 \%$ agar and then the plates were incubated at $37^{\circ} \mathrm{C}$.

\section{NPN uptake assay.}

The NPN uptake assay was conducted as described previously (Loh et al. 1984). Bacteria cultured in M9 minimal medium broth were washed twice in the assay buffer containing 
$5 \mathrm{mM}$ HEPES (pH 7.2) and $137 \mathrm{mM} \mathrm{NaCl}$ and resuspended in the assay buffer at $\mathrm{OD}_{600}=1.0$. The suspension $(100 \mu \mathrm{l})$ was mixed with $100 \mu$ l of NPN (Sigma-Aldrich) solution $(20 \mu \mathrm{M}$ in the assay buffer) in black 96-well plates (SPL Life Sciences, Gyeonggi-do, Korea), and NPN accumulation was immediately measured using the Cytation 3 Multi-Mode Reader (Bio-Tek, Winooski, VT, U.S.A.) at excitation and emission wavelengths of 350 and $420 \mathrm{~nm}$, respectively, every $30 \mathrm{~s}$ for $10 \mathrm{~min}$. Controls containing only NPN in the buffer were included for background subtraction, and the absorbance values were normalized using cell numbers.

\section{Bacterial resistance against acids, hydrogen peroxide, and sodium hypochlorite treatments.}

Bacteria at stationary phase in M9 broth were washed with PBS twice and resuspended in $\mathrm{ddH}_{2} \mathrm{O}$ at $\mathrm{OD}_{600}=0.5$. The bacterial suspension was treated with citric acid (1.5\%), lactic acid $(0.1 \%)$, hydrogen peroxide $(20 \mathrm{mM})$, and sodium hypochlorite $(0.05 \mathrm{mM})$ for $20 \mathrm{~min}$ and the viable cells were counted by plating on LB agar.

\section{Statistical analysis.}

All assays were repeated at least three times independently and the averaged values were presented with their standard deviations. Student's $t$ test was used to acquire $P$ values in determining the significance.

\section{LITERATURE CITED}

Ahmad, I., Lamprokostopoulou, A., Le Guyon, S., Streck, E., Barthel, M., Peters, V., Hardt, W. D., and Römling, U. 2011. Complex c-di-GMP signaling networks mediate transition between virulence properties and biofilm formation in Salmonella enterica serovar Typhimurium. PLoS One 6:e28351.

Barak, J. D., Jahn, C. E., Gibson, D. L., and Charkowski, A. O. 2007. The role of cellulose and $\mathrm{O}$-antigen capsule in the colonization of plants by Salmonella enterica. Mol. Plant-Microbe Interact. 20:1083-1091.

Berger, C. N., Shaw, R. K., Brown, D. J., Mather, H., Clare, S., Dougan, G., Pallen, M. J., and Frankel, G. 2009. Interaction of Salmonella enterica with basil and other salad leaves. ISME J. 3:261-265.

Brown, P. K., Dozois, C. M., Nickerson, C. A., Zuppardo, A., Terlonge, J., and Curtiss, R., 3rd. 2001. MlrA, a novel regulator of curli (AgF) and extracellular matrix synthesis by Escherichia coli and Salmonella enterica serovar Typhimurium. Mol. Microbiol. 41:349-363.

Chambers, J. R., and Sauer, K. 2013. Small RNAs and their role in biofilm formation. Trends Microbiol. 21:39-49.

Danese, P. N., Pratt, L. A., and Kolter, R. 2000. Exopolysaccharide production is required for development of Escherichia coli K-12 biofilm architecture. J. Bacteriol. 182:3593-3596.

Datsenko, K. A., and Wanner, B. L. 2000. One-step inactivation of chromosomal genes in Escherichia coli K-12 using PCR products. Proc. Natl. Acad. Sci. U.S.A. 97:6640-6645.

Deering, A. J., Mauer, L. J., and Pruitt, R. E. 2012. Internalization of E. coli O157:H7 and Salmonella spp. in plants: A review. Food Res. Int. 45: 567-575.

Deng, K., Wang, S., Rui, X., Zhang, W., and Tortorello, M. L. 2011. Functional analysis of $y c f R$ and $y c f Q$ in Escherichia coli O157:H7 linked to outbreaks of illness associated with fresh produce. Appl. Environ. Microbiol. 77:3952-3959.

Fornefeld, E., Schierstaedt, J., Jechalke, S., Grosch, R., Schikora, A., and Smalla K. 2017. Persistence of Salmonella Typhimurium LT2 in Soil Enhanced after Growth in Lettuce Medium. Front. Microbiol. 8:757.

Gerstel, U., Park, C., and Römling, U. 2003. Complex regulation of $\operatorname{csg} D$ promoter activity by global regulatory proteins. Mol. Microbiol. 49: 639-654.

Gómez Zavaglia, A., Kociubinski, G., Pérez, P., Disalvo, E., and De Antoni, G. 2002. Effect of bile on the lipid composition and surface properties of bifidobacteria. J. Appl. Microbiol. 93:794-799.

Hall-Stoodley, L., Costerton, J. W., and Stoodley, P. 2004. Bacterial biofilms: From the natural environment to infectious diseases. Nat. Rev. Microbiol. 2:95-108.

Kader, A., Simm, R., Gerstel, U., Morr, M., and Römling, U. 2006. Hierarchical involvement of various GGDEF domain proteins in rdar morphotype development of Salmonella enterica serovar Typhimurium Mol. Microbiol. 60:602-616.

Kisluk, G., and Yaron, S. 2012. Presence and persistence of Salmonella enterica serotype typhimurium in the phyllosphere and rhizosphere of spray-irrigated parsley. Appl. Environ. Microbiol. 78:4030-4036.

Lago, M., Monteil, V., Douche, T., Guglielmini, J., Criscuolo, A., Maufrais, C., Matondo, M., and Norel, F. 2017. Proteome remodelling by the stress sigma factor RpoS $/ \sigma^{\mathrm{S}}$ in Salmonella: Identification of small proteins and evidence for post-transcriptional regulation. Sci. Rep. 7: Article 2127.

Lapidot, A., Romling, U., and Yaron, S. 2006. Biofilm formation and the survival of Salmonella Typhimurium on parsley. Int. J. Food Microbiol. 109:229-233

Lee, I. S., Lin, J., Hall, H. K., Bearson, B., and Foster, J. W. 1995. The stationary-phase sigma factor $\sigma^{\mathrm{S}}(\mathrm{RpoS})$ is required for a sustained acid tolerance response in virulent Salmonella typhimurium. Mol. Microbiol. 17:155-167.

Lévi-Meyrueis, C., Monteil, V., Sismeiro, O., Dillies, M. A., Monot, M., Jagla, B., Coppée, J. Y., Dupuy, B., and Norel, F. 2014. Expanding the RpoS/ $\sigma^{\mathrm{S}}$-network by RNA sequencing and identification of $\sigma^{\mathrm{S}}$ controlled small RNAs in Salmonella. PLoS One 9:e96918.

Loh, B., Grant, C., and Hancock, R. E. 1984. Use of the fluorescent probe 1-N-phenylnaphthylamine to study the interactions of aminoglycoside antibiotics with the outer membrane of Pseudomonas aeruginosa. Antimicrob. Agents Chemother. 26:546-551.

Mah, T. F., and O'Toole, G. A. 2001. Mechanisms of biofilm resistance to antimicrobial agents. Trends Microbiol. 9:34-39.

Markland, S. M., Shortlidge, K. L., Hoover, D. G., Yaron, S., Patel, J., Singh, A., Sharma, M., and Kniel, K. E. 2013. Survival of pathogenic Escherichia coli on basil, lettuce, and spinach. Zoonoses Public Health 60:563-571.

Mayer, R., Ross, P., Weinhouse, H., Amikam, D., Volman, G., Ohana, P., Calhoon, R. D., Wong, H. C., Emerick, A. W., and Benziman, M. 1991. Polypeptide composition of bacterial cyclic diguanylic acid-dependent cellulose synthase and the occurrence of immunologically crossreacting proteins in higher plants. Proc. Natl. Acad. Sci. U.S.A. 88:5472-5476.

Morgan, J. L., McNamara, J. T., and Zimmer, J. 2014. Mechanism of activation of bacterial cellulose synthase by cyclic di-GMP. Nat. Struct. Mol. Biol. 21:489-496.

Ochman, H., and Groisman, E. A. 1995. The evolution of invasion by enteric bacteria. Can. J. Microbiol. 41:555-561.

Paul, K., Nieto, V., Carlquist, W. C., Blair, D. F., and Harshey, R. M. 2010. The c-di-GMP binding protein YcgR controls flagellar motor direction and speed to affect chemotaxis by a "backstop brake" mechanism. Mol. Cell 38:128-139.

Paytubi, S., Cansado, C., Madrid, C., and Balsalobre, C. 2017. Nutrient composition promotes switching between pellicle and bottom biofilm in Salmonella. Front. Microbiol. 8:2160.

Povolotsky, T. L., and Hengge, R. 2012. 'Life-style' control networks in Escherichia coli: Signaling by the second messenger c-di-GMP. J. Biotechnol. 160:10-16.

Rahman, M. M., Kim, W.-S., Kumura, H., and Shimazaki, K.-i. 2008. Autoaggregation and surface hydrophobicity of bifidobacteria. World J. Microbiol. Biotechnol. 24:1593-1598.

Raybaudi-Massilia, R. M., Mosqueda-Melgar, J., Soliva-Fortuny, R., and Martín-Belloso, O. 2009. Control of pathogenic and spoilage microorganisms in fresh-cut fruits and fruit juices by traditional and alternative natural antimicrobials. Compr. Rev. Food Sci. Food Saf. 8:157-180.

Rico, D., Martín-Diana, A. B., Barat, J. M., and Barry-Ryan, C. 2007. Extending and measuring the quality of fresh-cut fruit and vegetables: A review. Trends Food Sci. Technol. 18:373-386.

Römling, U. 2005. Characterization of the rdar morphotype, a multicellular behaviour in Enterobacteriaceae. Cell. Mol. Life Sci. 62:1234-1246.

Römling, U., Bian, Z., Hammar, M., Sierralta, W. D., and Normark, S. 1998. Curli fibers are highly conserved between Salmonella typhimurium and Escherichia coli with respect to operon structure and regulation. J. Bacteriol. 180:722-731.

Roy, A. B., Petrova, O. E., and Sauer, K. 2013. Extraction and quantification of cyclic Di-GMP from Pseudomonas aeruginosa. Bio-Protoc. 3:e828.

Salazar, J. K., Deng, K., Tortorello, M. L., Brandl, M. T., Wang, H., and Zhang, W. 2013. Genes $y c f R$, sirA and $y i g G$ contribute to the surface attachment of Salmonella enterica Typhimurium and Saintpaul to fresh produce. PLoS One 8:e57272.

Scher, K., Romling, U., and Yaron, S. 2005. Effect of heat, acidification, and chlorination on Salmonella enterica serovar typhimurium cells in a biofilm formed at the air-liquid interface. Appl. Environ. Microbiol. 71: 1163-1168.

Schikora, A., Garcia, A. V., and Hirt, H. 2012. Plants as alternative hosts for Salmonella. Trends Plant Sci. 17:245-249. 
Schmieger, H. 1972. Phage P22-mutants with increased or decreased transduction abilities. Mol. Gen. Genet. 119:75-88.

Shaw, R. K., Lasa, I., García, B. M., Pallen, M. J., Hinton, J. C., Berger, C. N., and Frankel, G. 2011. Cellulose mediates attachment of Salmonella enterica Serovar Typhimurium to tomatoes. Environ. Microbiol. Rep. 3:569-573.

Simm, R., Morr, M., Kader, A., Nimtz, M., and Römling, U. 2004. GGDEF and EAL domains inversely regulate cyclic di-GMP levels and transition from sessility to motility. Mol. Microbiol. 53:1123-1134.

Simm, R., Morr, M., Remminghorst, U., Andersson, M., and Römling, U. 2009. Quantitative determination of cyclic diguanosine monophosphate concentrations in nucleotide extracts of bacteria by matrix-assisted laser desorption/ionization-time-of-flight mass spectrometry. Anal. Biochem. 386:53-58.

Steenackers, H., Hermans, K., Vanderleyden, J., and De Keersmaecker, S. C. J. 2012. Salmonella biofilms: An overview on occurrence, structure, regulation and eradication. Food. Res. Int. 45:502-531.

Swords, W. E., Cannon, B. M., and Benjamin, W. H., Jr. 1997. Avirulence of LT2 strains of Salmonella typhimurium results from a defective rpoS gene. Infect. Immun. 65:2451-2453.

Uchiyama, J., Nobue, Y., Zhao, H., Matsuzaki, H., Nagahama, H., Matsuoka, S., Matsumoto, K., and Hara, H. 2010. Involvement of $\sigma^{\mathrm{s}}$ accumulation in repression of the flhDC operon in acidic phospholipiddeficient mutants of Escherichia coli. Microbiology 156:1650-1660.
Weber, H., Pesavento, C., Possling, A., Tischendorf, G., and Hengge, R. 2006. Cyclic-di-GMP-mediated signalling within the sigma network of Escherichia coli. Mol. Microbiol. 62:1014-1034.

Whitfield, C., and Trent, M. S. 2014. Biosynthesis and export of bacterial lipopolysaccharides. Annu. Rev. Biochem. 83:99-128.

Wilmes-Riesenberg, M. R., Foster, J. W., and Curtiss, R., 3rd. 1997. An altered rpos allele contributes to the avirulence of Salmonella typhimurium LT2. Infect. Immun. 65:203-210.

Xicohtencatl-Cortes, J., Sánchez Chacón, E., Saldaña, Z., Freer, E., and Girón, J. A. 2009. Interaction of Escherichia coli O157:H7 with leafy green produce. J. Food Prot. 72:1531-1537.

Yaron, S., and Römling, U. 2014. Biofilm formation by enteric pathogens and its role in plant colonization and persistence. Microb. Biotechnol. 7: 496-516.

Zhang, G., Meredith, T. C., and Kahne, D. 2013. On the essentiality of lipopolysaccharide to Gram-negative bacteria. Curr. Opin. Microbiol. 16:779-785.

Zhang, X.-S., García-Contreras, R., and Wood, T. K. 2007. YcfR (BhsA) influences Escherichia coli biofilm formation through stress response and surface hydrophobicity. J. Bacteriol. 189:3051-3062.

Zheng, J., Allard, S., Reynolds, S., Millner, P., Arce, G., Blodgett, R. J., and Brown, E. W. 2013. Colonization and internalization of Salmonella enterica in tomato plants. Appl. Environ. Microbiol. 79: 2494-2502. 